\title{
Non-resonant Alfvénic instability activated by high temperature of ion beams in compensated-current astrophysical plasmas
}

\author{
P. Malovichko' ${ }^{1}$ Y. Voitenko ${ }^{2}$, and J. De Keyser ${ }^{2}$ \\ 1 Main Astronomical Observatory, NASU, Kyiv, Ukraine \\ e-mail: malovichp@i.ua \\ 2 Solar-Terrestrial Centre of Excellence, Royal Belgian Institute for Space Aeronomy, Ringlaan 3, 1180 Brussels, Belgium \\ e-mail: voitenko@oma.be
}

Received 3 August 2017 / Accepted 23 April 2018

\begin{abstract}
Context. Compensated-current systems are established in response to hot ion beams in terrestrial foreshock regions, around supernova remnants, and in other space and astrophysical plasmas.

Aims. We study a non-resonant reactive instability of Alfvén waves propagating quasi-parallel to the background magnetic field $\boldsymbol{B}_{0}$ in such systems.

Methods. The instability is investigated analytically in the framework of kinetic theory applied to the hydrogen plasmas penetrated by hot proton beams.

Results. The instability arises at parallel wavenumbers $k_{\mathrm{z}}$ that are sufficiently large to demagnetize the beam ions, $k_{\mathrm{z}} V_{\mathrm{Tb}} / \omega_{\mathrm{Bi}} \gtrsim 1$ (here $V_{\mathrm{Tb}}$ is the beam thermal speed along $\boldsymbol{B}_{0}$ and $\omega_{\mathrm{Bi}}$ is the ion-cyclotron frequency). The Alfvén mode is then made unstable by the imbalance of perturbed currents carried by the magnetized background electrons and partially demagnetized beam ions. The destabilizing effects of the beam temperature and the temperature dependence of the instability threshold and growth rate are demonstrated for the first time. The beam temperature, density, and bulk speed are all destabilizing and can be combined in a single destabilizing factor $\alpha_{\mathrm{b}}$ triggering the instability at $\alpha_{\mathrm{b}}>\alpha_{\mathrm{b}}^{\text {thr }}$, where the threshold value varies in a narrow range $2.43 \leq \alpha_{\mathrm{b}}^{\text {thr }} \leq 4.87$. New analytical expressions for the instability growth rate and its boundary in the parameter space are obtained and can be directly compared with observations. Two applications to terrestrial foreshocks and foreshocks around supernova remnants are briefly discussed. In particular, our results suggest that the ions reflected by the shocks around supernova remnants can drive stronger instability than the cosmic rays.
\end{abstract}

Key words. plasmas - waves - instabilities - solar wind - ISM: supernova remnants

\section{Introduction}

Diluted ion beams propagating along the background magnetic field $\boldsymbol{B}_{0}$ are widespread in space and astrophysical plasmas, including solar wind (Marsch 2006, and references therein), terrestrial foreshocks (Paschmann et al. 1981, and references therein), supernova remnants (Bell 2005, and references therein), and many other astrophysical environments (Zweibel \& Everett 2010, and references therein). As the plasmas are typically quasi-neutral, the background electrons tend to follow the beam ions compensating their current. Depending on particular settings, the compensating currents can also be provided by other plasma components, like co-streaming electron beams injected simultaneously with the ion beams. Plasma instabilities developing in such compensated-current systems not only regulate the plasma and beam parameters keeping them close to the marginally unstable states, but can also be important sources for the background plasma heating, energetic particle acceleration, and amplification of the background magnetic field.

Plasma waves in the compensated-current systems can be driven unstable by resonant (Duijveman et al. 1981; Gary 1985; Vojtenko et al. 1990) and non-resonant (Winske \& Leroy 1984; Bell 2004; Achterberg 2013) wave-particle interactions. Resonant kinetic instabilities of various wave modes, driven by the beam ions, have been studied extensively in the past. Parallel-propagating Alfv én and fast waves have been found to be strongly unstable for beam velocities higher than a few Alfvén velocities (e.g., Gary 2005; Marsch 2006, and references therein). Concurrent instabilities of oblique (kinetic) Alfvén waves come into play at lower (but still super-Alfvénic) beam velocities (Voitenko 1998).

The above-mentioned instabilities can be driven directly by the beam ions (Sentman et al. 1981; Winske \& Leroy 1984; Gary 1985) or by the electron return currents (Winske \& Leroy 1984; Bell 2004; Chen \& Wu 2012, and references therein). The non-compensated electron currents flowing along $\boldsymbol{B}_{0}$, may also drive both the resonant (Voitenko 1995) and non-resonant (Malovichko \& Iukhimuk 1992; Malovichko 2007) instabilities of Alfvén waves. The simplest case of purely parallel propagating Alfvén waves has been considered in application to the current-carrying coronal loops (Malovichko \& Iukhimuk 1992), where these waves appeared to always be unstable. Later on, the analysis was extended by accounting for the oblique propagation (Malovichko 2007) and the currents curried by low-density beams (Malovichko 2010), and applied to the terrestrial magnetosphere and coronal loops.

Self-consistent modifications of the background magnetic field by the electric currents, neglected by Malovichko \& Iukhimuk (1992), Malovichko (2007); and Voitenko (1995), may reduce or even stabilize current instabilities. This issue does not concern instabilities developing in compensated-current systems. Such systems, formed around 
supernova remnants by high-energy streaming cosmic rays (CRs), were studied by Bell (2004), who found a new nonresonant Alfvénic instability (hereafter Bell instability). Since then, the Bell instability and its modifications have attracted a lot of interest (see, e.g., Amato \& Blasi 2009; Bret 2009; Zweibel \& Everett 2010; Schure et al. 2012; Achterberg 2013; Kobzar et al. 2017, and references therein). Following Bell (2004), the primary focus was on the unstable modes with finite $k_{\mathrm{z}} \bar{V}_{\mathrm{bz}} / \omega_{\mathrm{Bi}}$ propagating along $B_{0}$ (where $\bar{V}_{\mathrm{bz}}$ is a characteristic velocity of the beam ions along the mean magnetic field $\boldsymbol{B}_{0} \| z$; $k_{\mathrm{z}}$ is the parallel wavenumber; and $\omega_{\mathrm{Bi}}$ is the ion-cyclotron frequency). Compensated currents can also drive an oblique Alfvén instability (Malovichko et al. 2014) for which the perpendicular wave dispersion due to finite $k_{\perp} V_{\mathrm{Tb} \perp} / \omega_{\mathrm{Bi}}$ is essential $\left(k_{\perp}\right.$ and $V_{\mathrm{Tb} \perp}$ are the perpendicular wavenumber and beam thermal velocity in the plane $\perp \boldsymbol{B}_{0}$ ).

Other electrostatic and electromagnetic instabilities may develop in compensated-current systems (see, e.g., Gary 2005; Bret 2009; Brown et al. 2013; Marcowith et al. 2016, and references therein). Which wave modes grow fastest critically depends on the beam and plasma parameters. In the case of cold diluted proton beams propagating along $B_{0}$, the electrostatic two-stream and Buneman instabilities are much faster than the electromagnetic Alfvénic instabilities (see, e.g., Fig. 44 in Bret et al. 2010). Nevertheless, as is noted by Bret et al. (2010), these electrostatic instabilities are quickly saturated, and then electromagnetic Alfvénic/Bell instabilities come into play. In the hot beam/plasma systems, where the two-stream/Buneman instabilities cannot develop, the electromagnetic Alfvénic/Bell instabilities dominate.

The Bell instability has a maximum growth rate $\gamma_{\mathrm{Bell}} \simeq 0.5 \bar{j}_{\mathrm{b}} \omega_{\mathrm{Bi}}$, where $\bar{j}_{\mathrm{b}}=n_{\mathrm{b}} V_{\mathrm{b}} /\left(n_{0} V_{\mathrm{A}}\right)$ is the beam current normalized by the Alfvén current. This maximum is attained at the parallel wavenumber $\left|k_{\mathrm{zm}}\right| V_{\mathrm{A}} / \omega_{\mathrm{Bi}}=0.5 \bar{j}_{\mathrm{b}}$ and the perpendicular wavenumber $k_{\perp}=0$. These expressions are exactly the same as for the instability studied earlier by Winske \& Leroy (1984) in application to the terrestrial foreshock. The difference is that the role of $\bar{V}_{\mathrm{bz}}$ in the setting considered by Winske \& Leroy (1984) is played by the bulk velocity of the beam $V_{\mathrm{b}}$ rather than the large velocity spread of CRs. Both the Winske-Leroy and Bell instabilities grow fastest when the wave vector $\boldsymbol{k}$ is parallel to $\boldsymbol{B}_{0}$; they are physically the same instability that can be named the compensated-current parallel instability (CCPI).

The physical mechanism of the CCPI is related to the fact that for sufficiently small parallel wavelengths and sufficiently high $\bar{V}_{\text {bz }}$, the beam protons become partially demagnetized (unfrozen off the perturbed magnetic field). The demagnetization reduces the beam contribution to the fluctuating currents $\delta \boldsymbol{j} \perp$ $\boldsymbol{B}_{0}$ flowing along the twisted perturbed magnetic field lines, whereas the electron currents remain magnetized, thus providing the non-compensated fluctuating transversal currents. These currents amplify the initial perturbations via the positive feedback loop giving rise to CCPI. This kind of instability is sometimes called reactive.

Surprisingly, despite its importance in astrophysical applications, the CCPI theory is still poorly developed. Many important properties of the instability (the wavenumber dependence of the instability growth rate, behavior of the maximum growth rate in the parameter space, instability boundaries in the parameter spaces, etc.) have not been fully investigated. In the present paper, we study CCPI of Alfvén waves in more detail in the framework of kinetic theory. We consider a simple model of the compensated-current system where the hydrogen plasma is hydrogen plasma is penetrated by the low-density proton beam and the beam current and charge are compensated by the background electrons. Despite its simplicity, this model is applicable to the reactive CCPI driven by compensated currents in many space and astrophysical environments.

\section{Plasma model and dispersion equation for Alfvén waves}

We consider a three-component plasma consisting of the background steady ion component (i), the low-density ion beam (b) propagating with velocity $V_{\mathrm{b}}$ along $z \| B_{0}$, and the electron component (e) providing the neutralizing current and charge:

$n_{\mathrm{e}} V_{\mathrm{e}}=n_{\mathrm{b}} V_{\mathrm{b}}$

$n_{\mathrm{e}}=n_{\mathrm{i}}+n_{\mathrm{b}} \equiv n_{0}$.

We assume here that the beam ions (b) and the background ions (i) are protons. All plasma components are modeled by the shifted Maxwellian velocity distributions

$f_{0 s}=\frac{n_{\mathrm{s}}}{(2 \pi)^{3 / 2} V_{\mathrm{Ts}}^{3}} \exp \left(-\frac{v_{\perp}^{2}}{V_{\mathrm{Ts}}^{2}}-\frac{\left(v_{z}-V_{\mathrm{s}}\right)^{2}}{V_{\mathrm{Ts}}^{2}}\right)$,

where $n_{\mathrm{s}}, V_{\mathrm{s}}, V_{\mathrm{Ts}}=\sqrt{T_{\mathrm{s}} / m_{\mathrm{s}}}, T_{\mathrm{s}}$, and $m_{\mathrm{s}}$ are the mean number density, parallel bulk velocity, thermal velocity, temperature, and particle mass of the plasma species $\mathrm{s}$, and $\boldsymbol{v}=\left(v_{\mathrm{x}}, v_{\mathrm{y}}, v_{\mathrm{z}}\right)-$ velocity-space coordinates. The subscripts $z$ and $\perp$ indicate directions parallel and perpendicular to $\boldsymbol{B}_{0}$. The plasma model defined by Eqs. ((1)-(3)) has been extensively used in the past (see, e.g., Gary 2005, and references therein). The neutralizing current can also be provided by the co-propagating electron beam (see, e.g., Zweibel \& Everett 2010 and references therein); however it does not alter the reactive CCPI for low-density ion beams $n_{\mathrm{b}} \ll n_{0}$ (Amato \& Blasi 2009).

The non-trivial solutions to the Maxwell-Vlasov set of equations exist if the wave frequency $\omega$ and the wave vector $\boldsymbol{k}=\left(k_{\mathrm{x}}, k_{\mathrm{y}}, k_{\mathrm{z}}\right)$ satisfy the following dispersion equation (see, e.g., Alexandrov et al. 1984)

$\left|k^{2} \delta_{\mathrm{ij}}-k_{\mathrm{i}} k_{\mathrm{j}}-\frac{\omega^{2}}{c^{2}} \varepsilon_{\mathrm{ij}}\right|=0$,

where $\varepsilon_{\mathrm{ij}}$ is the dielectric tensor and $\delta_{\mathrm{ij}}$ is the Kronecker delta. For the parallel-propagating modes with $k_{\mathrm{x}}=k_{\mathrm{y}}=0$, the components of the dielectric tensor given by Alexandrov et al. (1984) reduce to

$\varepsilon_{\mathrm{xx}}=\varepsilon_{\mathrm{yy}}=1-\sum_{\mathrm{s}}\left(\frac{\omega_{\mathrm{Ps}}}{\omega}\right)^{2} \frac{1}{2} \sum_{n= \pm 1} \frac{\xi_{\mathrm{s}, 0}}{\xi_{\mathrm{s}, \mathrm{n}}} J_{+}\left(\xi_{\mathrm{s}, \mathrm{n}}\right) ;$

$\varepsilon_{\mathrm{xy}}=-\varepsilon_{\mathrm{yx}}=i \sum_{\mathrm{s}}\left(\frac{\omega_{\mathrm{Ps}}}{\omega}\right)^{2} \frac{1}{2} \sum_{n= \pm 1} n \frac{\xi_{\mathrm{s}, 0}}{\xi_{\mathrm{s}, \mathrm{n}}} J_{+}\left(\xi_{\mathrm{s}, \mathrm{n}}\right) ;$

$\varepsilon_{\mathrm{xz}}=\varepsilon_{\mathrm{zx}}=\varepsilon_{\mathrm{yz}}=\varepsilon_{\mathrm{zy}}=0$;

$\varepsilon_{\mathrm{zz}}=1+\sum_{\mathrm{s}}\left(\frac{\omega_{\mathrm{Ps}}}{k_{\mathrm{z}} V_{\mathrm{Ts}}}\right)^{2}\left[1-J_{+}\left(\xi_{\mathrm{s}, 0}\right)\right]$,

where $\xi_{\mathrm{s}, \mathrm{n}}=\left(\omega-k_{\mathrm{z}} V_{\mathrm{s}}+n \omega_{\mathrm{Bs}}\right) /\left(k_{\mathrm{z}} V_{\mathrm{Ts}}\right), \omega_{\mathrm{Ps}}\left(\omega_{\mathrm{Bs}}\right)$ is the plasma (cyclotron) frequency. Instead of the plasma dispersion function $W(x)$, we use the function

$J_{+}(x)=-i \sqrt{\frac{\pi}{2}} x W\left(\frac{x}{\sqrt{2}}\right)=x \exp \left(-\frac{x^{2}}{2}\right) \int_{i \infty}^{x} \mathrm{~d} t \exp \left(\frac{t^{2}}{2}\right)$, 
introduced by Alexandrov et al. (1984). It has the following asymptotic expansions:

$J_{+}(x)=x^{2}+O\left(x^{4}\right)-i \sqrt{\frac{\pi}{2}} x \exp \left(-\frac{x^{2}}{2}\right), \quad|x| \ll 1 ;$

and

$J_{+}(x)=1+\frac{1}{x^{2}}+O\left(\frac{1}{x^{4}}\right)-i \eta \sqrt{\frac{\pi}{2}} x \exp \left(-\frac{x^{2}}{2}\right), \quad|x| \gg 1$,

where $\eta=0$ for $\operatorname{Im} x>0, \eta=1$ for $\operatorname{Im} x=0$, and $\eta=2$ for $\operatorname{Im} x<0$.

In the case of parallel propagation, the dispersion Eq. (4) splits into two independent equations,

$\varepsilon_{\mathrm{xx}} \pm i \varepsilon_{\mathrm{xy}}=\left(\frac{c k_{\mathrm{z}}}{\omega}\right)^{2}$,

describing left-hand (sign -) and right-hand (sign +) polarized electromagnetic waves. In what follows we consider the left-hand polarized Alfvén branch undergoing the compensatedcurrent instability. Taking into account quasi-neutrality (2) and current compensation (1), Eq. (9) for Alfvén waves can be written as

$\left(\frac{\omega}{\omega_{\mathrm{Bi}}}\right)^{2}-\frac{n_{\mathrm{b}}}{n_{0}} A_{\mathrm{k}, \omega} \frac{\omega}{\omega_{\mathrm{Bi}}}-\left(\frac{k_{\mathrm{z}} V_{\mathrm{A}}}{\omega_{\mathrm{Bi}}}\right)^{2}+\frac{n_{\mathrm{b}}}{n_{0}} A_{\mathrm{k}, \omega} \frac{k_{\mathrm{z}} V_{\mathrm{b}}}{\omega_{\mathrm{Bi}}}=0$,

where

$A_{\mathrm{k}, \omega}=1+\frac{\omega_{\mathrm{Bi}}}{k_{\mathrm{z}} V_{\mathrm{Tb}}} \frac{J_{+}\left(\xi_{\mathrm{b},-1}\right)}{\xi_{\mathrm{b},-1}}$.

In the following sections we consider important limits of (10) typical for the reactive CCPI instability.

\section{Dispersion relation for parallel-propagating waves}

As we are going to analyze the reactive non-resonant instability, we neglect the contribution of the imaginary part of $J_{+}\left(\xi_{\mathrm{b},-1}\right)$. Furthermore, we consider a low-frequency instability with $\left|\omega / \omega_{\mathrm{Bi}}\right|$ smaller than other terms in $\xi_{\mathrm{b},-1}$, which allows us to neglect the $\omega$-dependent part in the argument of function $J_{+}$. In this case (10) reduces to the following quadratic equation with respect to $\omega / \omega_{\mathrm{Bi}}$ :

$\left(\frac{\omega}{\omega_{\mathrm{Bi}}}\right)^{2}-\frac{n_{\mathrm{b}}}{n_{0}} A_{\mathrm{k}} \frac{\omega}{\omega_{\mathrm{Bi}}}-\left(\frac{k_{\mathrm{z}} V_{\mathrm{A}}}{\omega_{\mathrm{Bi}}}\right)^{2}+\frac{n_{\mathrm{b}}}{n_{0}} A_{\mathrm{k}} \frac{k_{\mathrm{z}} V_{\mathrm{b}}}{\omega_{\mathrm{Bi}}}=0$,

where

$A_{\mathrm{k}} \equiv A_{\mathrm{k}, 0}=1+\frac{1}{k_{\mathrm{z}} \rho_{\mathrm{Tb}}} \frac{\operatorname{Re} J_{+}\left(\zeta_{\mathrm{b}}\right)}{\zeta_{\mathrm{b}}}$,

$\zeta_{\mathrm{b}}=-V_{\mathrm{b}} / V_{\mathrm{Tb}}-1 /\left(k_{\mathrm{z}} \rho_{\mathrm{Tb}}\right)$, and $\rho_{\mathrm{Tb}}=V_{\mathrm{Tb}} / \omega_{\mathrm{Bi}}$. To avoid misunderstanding, we note that although $\rho_{\mathrm{Tb}}$ looks like the ion beam gyroradius, it is defined by the parallel beam temperature rather than the perpendicular one, and here it has a different physical meaning.

Equation (11) is the second-order eigenmode equation for Alfvén waves modified by the ion beam and return electron current (second and fourth terms, respectively). Its solution is straightforward:

$\frac{\omega}{\omega_{\mathrm{Bi}}}=\frac{n_{\mathrm{b}}}{n_{0}} \frac{A_{\mathrm{k}}}{2}+\sqrt{\left(\frac{n_{\mathrm{b}}}{n_{0}} \frac{A_{\mathrm{k}}}{2}\right)^{2}+\left(\frac{k_{\mathrm{z}} V_{\mathrm{A}}}{\omega_{\mathrm{Bi}}}\right)^{2}-2 \frac{n_{\mathrm{b}}}{n_{0}} \frac{A_{\mathrm{k}}}{2} \frac{k_{\mathrm{z}} V_{\mathrm{b}}}{\omega_{\mathrm{Bi}}} .}$
From (13) it is obvious that the instability can be driven by the last term under the square root when $k_{\mathrm{z}} V_{\mathrm{b}}>0$. In what follows we assume $V_{\mathrm{b}}>0$ considering potentially unstable waves with $k_{\mathrm{z}}>0$ (in the case of $V_{\mathrm{b}}<0$, the identical instability develops for $\left.k_{\mathrm{z}}<0\right)$. In the absence of the beam, Eq. (13) reduces to the Alfvén wave dispersion, $\omega=k_{\mathrm{z}} V_{\mathrm{A}}$ at $n_{\mathrm{b}}=0$.

The wave with dispersion (13) becomes unstable when the last term under the square root dominates. This term represents effects due to the electron current. The growth rate $\gamma=\operatorname{Im}[\omega]$ of the corresponding instability is

$\frac{\gamma_{\mathrm{k}}}{\omega_{\mathrm{Bi}}}=\frac{V_{\mathrm{A}}}{V_{\mathrm{Tb}}} \sqrt{2 k_{\mathrm{z}} \rho_{\mathrm{Tb}} \frac{\alpha_{\mathrm{b}} A_{\mathrm{k}}}{2}-\left(k_{\mathrm{z}} \rho_{\mathrm{Tb}}\right)^{2}-\left(\frac{V_{\mathrm{A}}}{V_{\mathrm{b}}}\right)^{2}\left(\frac{\alpha_{\mathrm{b}} A_{\mathrm{k}}}{2}\right)^{2}}$.

Here we introduce the cumulative destabilizing parameter

$\alpha_{\mathrm{b}}=\frac{n_{\mathrm{b}}}{n_{0}} \frac{V_{\mathrm{b}}}{V_{\mathrm{A}}} \frac{V_{\mathrm{Tb}}}{V_{\mathrm{A}}} \equiv \bar{j}_{\mathrm{b}} \bar{V}_{\mathrm{Tb}}$

that includes all beam parameters. One can think of it as of product of the normalized current $\bar{j}_{\mathrm{b}}=n_{\mathrm{b}} V_{\mathrm{b}} /\left(n_{0} V_{\mathrm{A}}\right)$ and velocity spread $\bar{V}_{\mathrm{Tb}}=V_{\mathrm{Tb}} / V_{\mathrm{A}}$ of the beam ions. The growth rate (14) is analyzed below analytically and numerically, and its scalings are found in some important limits. It is interesting to note that the right-hand polarized magnetosonic instability can be obtained from the above equation by changing the sign of the first term under the square root (the magnetosonic instability hence requires $\left.k_{\mathrm{z}} V_{\mathrm{b}}<0\right)$.

\section{Compensated-current instability driven by hot ion beams}

By hot beams we mean the beams whose thermal velocity is significantly larger than the bulk velocity, $V_{\mathrm{Tb}} \gg V_{\mathrm{b}}$. For such beams, the growth rate (14) can be simplified by neglecting the small term $V_{\mathrm{b}} / V_{\mathrm{Tb}}$ in $\xi_{\mathrm{b},-1}$. The argument of $J_{+}$is then simplified to $\xi_{\mathrm{b},-1} \approx-1 /\left(k_{\mathrm{z}} \rho_{\mathrm{Tb}}\right) \equiv \zeta_{\mathrm{b}}$. In this case $\gamma_{\mathrm{k}}$ depends on the normalized parallel wavenumber $k_{\mathrm{z}} \rho_{\mathrm{Tb}}$ and two dimensionless bulk parameters, $V_{\mathrm{A}} / V_{\mathrm{b}}$ and $\alpha_{\mathrm{b}}$. Then the (maximum) instability growth rate $\gamma_{\mathrm{m}}=\max _{k} \gamma$ appears to be a function of $\alpha_{\mathrm{b}}$ and $V_{\mathrm{A}} / V_{\mathrm{b}}$ only, whereas the dependence on the general multiplier $V_{\mathrm{A}} / V_{\mathrm{Tb}}$ is trivial and can be excluded by the renormalization of $\gamma_{\mathrm{m}}$. We note that the hot-beam condition $V_{\mathrm{Tb}}>V_{\mathrm{b}}$ restricts the applicability range of the analytical results obtained below, but in general it does not restrict the instability range (see also Sect. 6).

\subsection{Instability areas in the parameter space}

Here we find the instability threshold and the instability area in the parameter space $\left(\alpha_{\mathrm{b}}, V_{\mathrm{A}} / V_{\mathrm{b}}\right)$. To this end, we present the growth rate (14) in the following useful form:

$\frac{\gamma_{\mathrm{k}}}{\omega_{\mathrm{Bi}}}=\frac{V_{\mathrm{b}}}{V_{\mathrm{Tb}}} k_{\mathrm{z}} \rho_{\mathrm{Tb}} \sqrt{1-\frac{V_{\mathrm{A}}^{2}}{V_{\mathrm{b}}^{2}}-\left(1-\frac{V_{\mathrm{A}}^{2}}{V_{\mathrm{b}}^{2}} \frac{\alpha_{\mathrm{b}}}{2} \frac{A_{\mathrm{k}}}{k_{\mathrm{z}} \rho_{\mathrm{Tb}}}\right)^{2}}$.

From (16), the instability condition is obtained as

$1-\frac{V_{\mathrm{A}}^{2}}{V_{\mathrm{b}}^{2}}>\left(1-\frac{V_{\mathrm{A}}^{2}}{V_{\mathrm{b}}^{2}} \frac{\alpha_{\mathrm{b}}}{2} \frac{A_{\mathrm{k}}}{k_{\mathrm{z}} \rho_{\mathrm{Tb}}}\right)^{2}$.

Since the right-hand side of (17) is positive, it is obvious that only super-Alfvén beams, $V_{\mathrm{b}}>V_{\mathrm{A}}$, can trigger instability. Therefore, the absolute threshold for the beam velocity is 
$V_{\mathrm{b}}^{\mathrm{thr}}=V_{\mathrm{A}}$ and the system is stable with respect to reactive CCPI for all $V_{\mathrm{b}}<V_{\mathrm{A}}$.

Using (17), it is also possible to find the threshold for $\alpha_{\mathrm{b}}$ analytically. First, solving (17) with respect to the $k_{k}$-dependent term $A_{\mathrm{k}} /\left(k_{\mathrm{z}} \rho_{\mathrm{Tb}}\right)$, we find that the unstable wavenumbers $k_{\mathrm{z}}$ should satisfy

$$
\frac{2}{\alpha_{\mathrm{b}}} \frac{1}{1+\sqrt{1-\frac{V_{\mathrm{A}}^{2}}{V_{\mathrm{b}}^{2}}}}<\frac{A_{\mathrm{k}}}{k_{\mathrm{z}} \rho_{\mathrm{Tb}}}<\frac{2}{\alpha_{\mathrm{b}}} \frac{V_{\mathrm{b}}^{2}}{V_{\mathrm{A}}^{2}}\left(1+\sqrt{1-\frac{V_{\mathrm{A}}^{2}}{V_{\mathrm{b}}^{2}}}\right) .
$$

When the velocity threshold is exceeded, $V_{\mathrm{b}}>V_{\mathrm{A}}$, the right boundary of (18) is always larger than the left boundary making the interval between them non-empty. As the function $A_{\mathrm{k}} /\left(k_{\mathrm{z}} \rho_{\mathrm{Tb}}\right)$ is limited by the maximum value $\max _{k}\left[A_{\mathrm{k}} /\left(k_{\mathrm{z}} \rho_{\mathrm{Tb}}\right)\right] \approx 0.411$ achieved at $k_{\mathrm{z}}^{*} \rho_{\mathrm{Tb}} \approx 1.541$, the condition (18) can only be satisfied for sufficiently large $\alpha_{\mathrm{b}}$. From the left-hand inequality, it immediately follows the instability condition for $\alpha_{\mathrm{b}}$ and the corresponding threshold:

$$
\alpha_{\mathrm{b}}>\alpha_{\mathrm{b}}^{\mathrm{thr}}=\frac{2}{\max _{k}\left[\frac{A_{\mathrm{k}}}{k_{\mathrm{z}} \rho_{\mathrm{Tb}}}\right]\left(1+\sqrt{1-\frac{V_{\mathrm{A}}^{2}}{V_{\mathrm{b}}^{2}}}\right)}=\frac{4.866}{1+\sqrt{1-\left(\frac{V_{\mathrm{A}}}{V_{\mathrm{b}}}\right)^{2}}} .
$$

The instability condition $\alpha_{\mathrm{b}}>\alpha_{\mathrm{b}}^{\mathrm{thr}}$ is satisfied above the threshold curve defined by (19), which is shown in Fig. 1 by the solid line. The unstable area above this curve in the parameter space $\left(\alpha_{\mathrm{b}}, V_{\mathrm{A}} / V_{\mathrm{b}}\right)$ is shaded. The dependence of the threshold $\alpha_{\mathrm{b}}^{\text {thr }}$ on $V_{\mathrm{A}} / V_{\mathrm{b}}$ is rather weak; it grows from the minimum value $\alpha_{\mathrm{b}}^{\text {thr }} \approx 2.43$ at $V_{\mathrm{A}} / V_{\mathrm{b}} \rightarrow 0$ to the maximum value $\alpha_{\mathrm{b}}^{\text {thr }} \approx 4.87$ at $V_{\mathrm{A}} / V_{\mathrm{b}} \rightarrow 1$. The absolute threshold for $\alpha_{\mathrm{b}}$ below which the system is stable is $\alpha_{\mathrm{bmin}}^{\mathrm{thr}} \approx 2.43$. The meaning of the right boundary in (18), shown in Fig. 1 by the dashed line, is clarified in the following subsection.

In terms of the normalized beam current $\bar{j}_{\mathrm{b}}=n_{\mathrm{b}} V_{\mathrm{b}} /\left(n_{0} V_{\mathrm{A}}\right)$ and thermal velocity $\bar{V}_{\mathrm{Tb}}=V_{\mathrm{Tb}} / V_{\mathrm{A}}$, (19) can be written as $\bar{j}_{\mathrm{b}} \bar{V}_{\mathrm{Tb}}>\alpha_{\mathrm{b}}^{\text {thr }}$. Then the instability condition for the beam thermal velocity reads as

$\bar{V}_{\mathrm{Tb}}>\bar{V}_{\mathrm{Tb}}^{\mathrm{thr}}=\frac{\alpha_{\mathrm{b}}^{\mathrm{thr}}}{\bar{j}_{\mathrm{b}}}$.

This threshold-like condition is an important new result quantitatively demonstrating the destabilizing effect of the beam temperature. It shows the threshold above which the velocity spread of the beam ions triggers the instability even for weak beams.

Similarly, the threshold condition for the beam current can be written as

$$
\bar{j}_{\mathrm{b}}>\bar{j}_{\mathrm{b}}^{\mathrm{thr}} \approx \frac{\alpha_{\mathrm{b}}^{\mathrm{thr}}}{\bar{V}_{\mathrm{Tb}}},
$$

which quantifies the range of unstable beam currents. Again, it is seen that even a weak ion beam can activate CCPI if the beam thermal velocity is sufficiently high. In particular, the beam current required for the instability can be many orders of magnitude smaller than the Alfvén current.

We note that $\alpha_{\mathrm{b}}^{\text {thr }}$ varies slowly for fast super-Alfv énic beams and can be approximated as $\alpha_{\mathrm{b}}^{\text {thr }} \approx 2.5$ at $V_{\mathrm{b}} / V_{\mathrm{A}}>3$. For rough estimations, in all velocity ranges $\alpha_{\mathrm{b}}^{\text {thr }}$ can be replaced by its average value 3.5 .

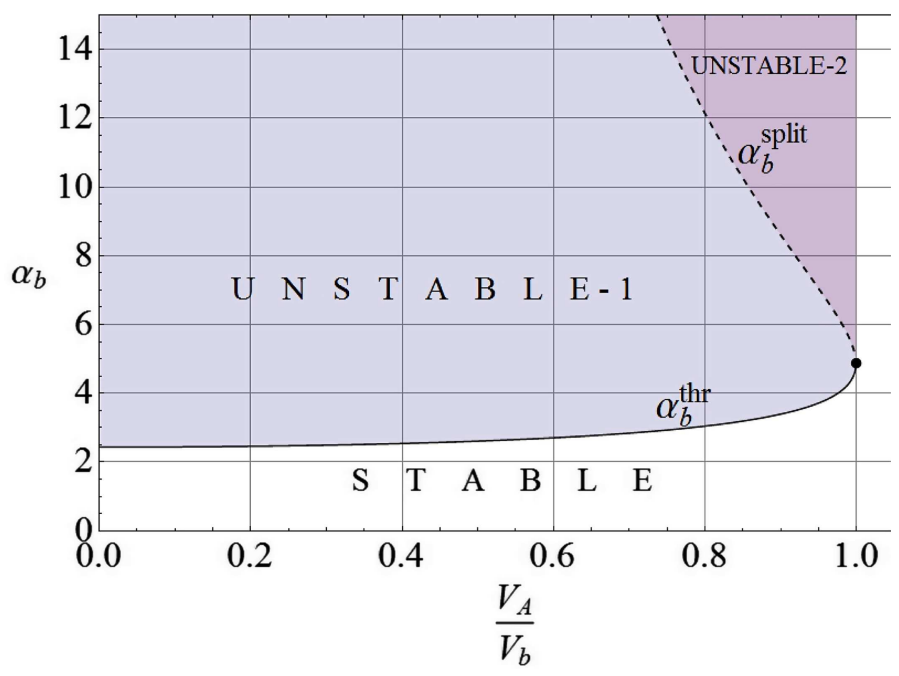

Fig. 1. Instability threshold $\alpha_{\mathrm{b}}^{\text {thr }}$ in the parameter space $\left(\alpha_{\mathrm{b}}, V_{\mathrm{A}} / V_{\mathrm{b}}\right)$ (solid line); the CCPI develops at all $\alpha_{\mathrm{b}}>\alpha_{\mathrm{b}}^{\text {thr }}$. The dashed line shows the split threshold $\alpha_{\mathrm{b} 2}^{\text {thr }}$ above which there are two separate ranges of unstable wavenumbers $k_{\mathrm{z}}$

\subsection{Unstable wavenumber ranges}

Properties of CCPI are illustrated further by Figs. 2 and 3 showing all three terms of the condition (18): the left and right boundaries, and the function $\left(k_{\mathrm{z}} \rho_{\mathrm{Tb}}\right)^{-1} A_{\mathrm{k}}$. The unstable ranges where (18) is satisfied are shaded. A regular single-peak behavior of the function $\left(k_{\mathrm{z}} \rho_{\mathrm{Tb}}\right)^{-1} A_{\mathrm{k}}$, as seen in Figs. 2 and 3 , allows us to investigate how the unstable wavenumber range evolves with $\alpha_{\mathrm{b}}$.

When $\alpha_{\mathrm{b}}$ increases but is still smaller than $\alpha_{\mathrm{b}}^{\text {thr }}$, the left boundary of (18) decreases remaining above the maximum of $\left(k_{\mathrm{z}} \rho_{\mathrm{Tb}}\right)^{-1} A_{\mathrm{k}}$. In this case there are no unstable wavenumbers and the system is stable. Once $\alpha_{\mathrm{b}}$ rises above $\alpha_{\mathrm{b}}^{\text {thr }}$, the decreasing left boundary of (18) drops below the maximum of $\left(k_{\mathrm{z}} \rho_{\mathrm{Tb}}\right)^{-1} A_{\mathrm{k}}$ and the unstable wavenumber range $k_{\mathrm{z} 1}<k_{\mathrm{z}}<k_{\mathrm{z} 2}$ appears, where $k_{\mathrm{z} 1}$ and $k_{\mathrm{z} 2}$ are the lower and upper roots of the equation

$\frac{A_{\mathrm{k}}}{k_{\mathrm{z}} \rho_{\mathrm{Tb}}}=\frac{2}{\alpha_{\mathrm{b}}} \frac{1}{1+\sqrt{1-\frac{V_{\mathrm{A}}^{2}}{V_{\mathrm{b}}^{2}}}}$.

As long as $\alpha_{\mathrm{b}}$ is not far from the threshold $\alpha_{\mathrm{b}}^{\mathrm{thr}}$, there is a single unstable wavenumber interval surrounding $k_{\mathrm{z}}^{*} \rho_{\mathrm{Tb}} \approx 1.54$. This situation is illustrated in Fig. 2, where $V_{\mathrm{A}} / V_{\mathrm{b}}=0.9, \alpha_{\mathrm{b}}^{\text {thr }} \approx 3.4$, and $\alpha_{\mathrm{b}}=6>\alpha_{\mathrm{b}}^{\text {thr }}$. However, when $\alpha_{\mathrm{b}}$ increases further, the right boundary of (18) also drops below the maximum of $\left(k_{\mathrm{z}} \rho_{\mathrm{Tb}}\right)^{-1} A_{\mathrm{k}}$, which happens at

$\alpha_{\mathrm{b}}>\alpha_{\mathrm{b}}^{\text {split }}=4.866 \frac{V_{\mathrm{b}}^{2}}{V_{\mathrm{A}}^{2}}\left(1+\sqrt{1-\frac{V_{\mathrm{A}}^{2}}{V_{\mathrm{b}}^{2}}}\right)$.

In this case, shown in Fig. 3 for $\alpha_{\mathrm{b}}=9$, the right-hand side inequality of (18) is not satisfied in the range $k_{\mathrm{z} 1}^{\prime}<k_{\mathrm{z}}<k_{\mathrm{z} 2}^{\prime}$, where $k_{\mathrm{z} 1}^{\prime}$ and $k_{\mathrm{z} 2}^{\prime}$ are the lower and upper roots of the equation

$\frac{A_{\mathrm{k}}}{k_{\mathrm{z}} \rho_{\mathrm{Tb}}}=\frac{2}{\alpha_{\mathrm{b}}} \frac{V_{\mathrm{b}}^{2}}{V_{\mathrm{A}}^{2}}\left(1+\sqrt{1-\frac{V_{\mathrm{A}}^{2}}{V_{\mathrm{b}}^{2}}}\right)$.

Instead of unstable, we have now a prohibited wavenumber range around $k_{\mathrm{z}}^{*} \rho_{\mathrm{Tb}} \approx 1.54$. As a result, the unstable wavenumber range splits into two: the first unstable range is $k_{\mathrm{z} 1}<k_{\mathrm{z}}<k_{\mathrm{z} 1}^{\prime}$ and the second $k_{\mathrm{z} 2}^{\prime}<k_{\mathrm{z}}<k_{\mathrm{z} 2}$. 
P. Malovichko et al.: Non-resonant Alfvénic instability activated by high beam temperature

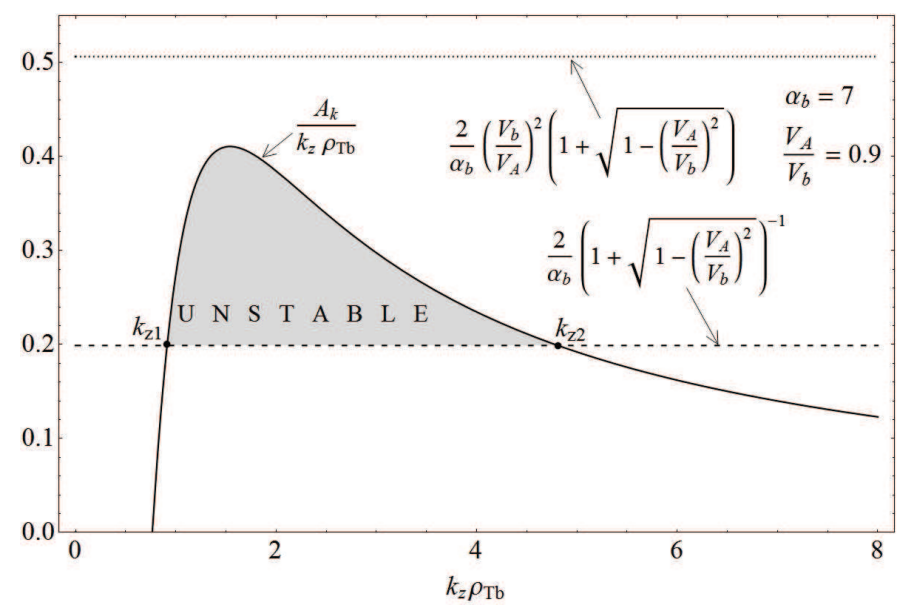

Fig. 2. Illustration of the condition (18) for $V_{\mathrm{A}} / V_{\mathrm{b}}=0.9$ and $\alpha_{\mathrm{b}}=6$. In this case $\alpha_{\mathrm{b}}^{\text {thr }}<\alpha_{\mathrm{b}}<\alpha_{\mathrm{b}}^{\text {split }}$ and there is only one unstable wavenumber range (shaded area).

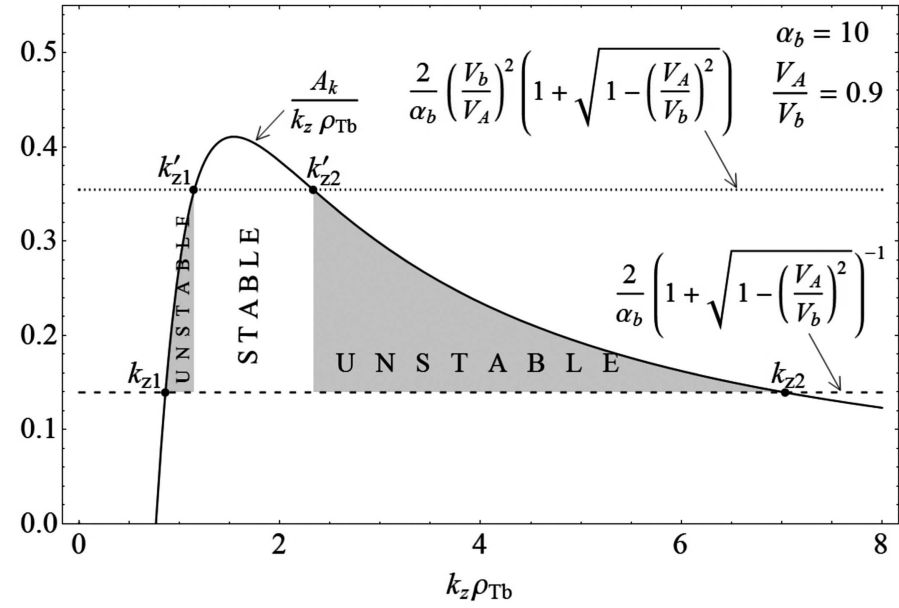

Fig. 3. Illustration of the condition (18) for $V_{\mathrm{A}} / V_{\mathrm{b}}=0.9$ and $\alpha_{\mathrm{b}}=10$ In this case $\alpha_{\mathrm{b}}>\alpha_{\mathrm{b}}^{\text {split }}$ and there two unstable wavenumber ranges presented by two shaded areas.

The split threshold $\alpha_{\mathrm{b}}^{\text {split }}(23)$ is shown in Fig. 1 by the dashed line. For parameter values above this line, the instability develops in two wavenumber ranges, as mentioned above. These unstable ranges are shown in Fig. 3 by the shaded areas.

Furthermore, Fig. 4 shows the $\alpha_{\mathrm{b}}$ dependence of the unstable wavenumber ranges, where the outer and inner boundaries are respectively defined by the left-hand and right-hand margins of (18). It is seen that below $\alpha_{\mathrm{b}}^{\text {thr }}$ there is no instability, at $\alpha_{\mathrm{b}}^{\text {thr }}<\alpha_{\mathrm{b}}<\alpha_{\mathrm{b}}^{\text {split }}$ there is a single unstable range of $k_{\mathrm{z}}$, and above $\alpha_{\mathrm{b}}^{\text {split }}$ there are two unstable ranges.

From Fig. 3 it is obvious that $k_{\mathrm{z} 1} \rho_{\mathrm{Tb}}$ and $k_{\mathrm{z} 1}^{\prime} \rho_{\mathrm{Tb}}$ are located between $k_{\mathrm{z}} \rho_{\mathrm{Tb}} \approx 0.77$, where $\left(k_{\mathrm{z}} \rho_{\mathrm{Tb}}\right)^{-1} A_{\mathrm{k}}$ is zero, and $k_{\mathrm{z}}^{*} \rho_{\mathrm{Tb}} \approx 1.54$, where $\left(k_{\mathrm{z}} \rho_{\mathrm{Tb}}\right)^{-1} A_{\mathrm{k}}$ is maximum. This wavenumber range corresponds to $-1.3<\zeta_{\mathrm{b}}<-0.65$, where $\operatorname{Re} J_{+}\left(\zeta_{\mathrm{b}}\right)$ can be approximated by the liner numerical fit

$\operatorname{Re} J_{+}\left(\zeta_{\mathrm{b}}\right) \approx-0.275-\zeta_{\mathrm{b}}$.

Using this in (22) and (24), we find $k_{\mathrm{z} 1}$ and $k_{\mathrm{z} 1}^{\prime}$ as

$k_{\mathrm{z} 1} \rho_{\mathrm{Tb}} \approx \frac{1}{0.64+\sqrt{0.41-\frac{2}{\alpha_{\mathrm{b}}}\left(1+\sqrt{1-\frac{V_{\mathrm{A}}^{2}}{V_{\mathrm{b}}^{2}}}\right)^{-1}}} ;$

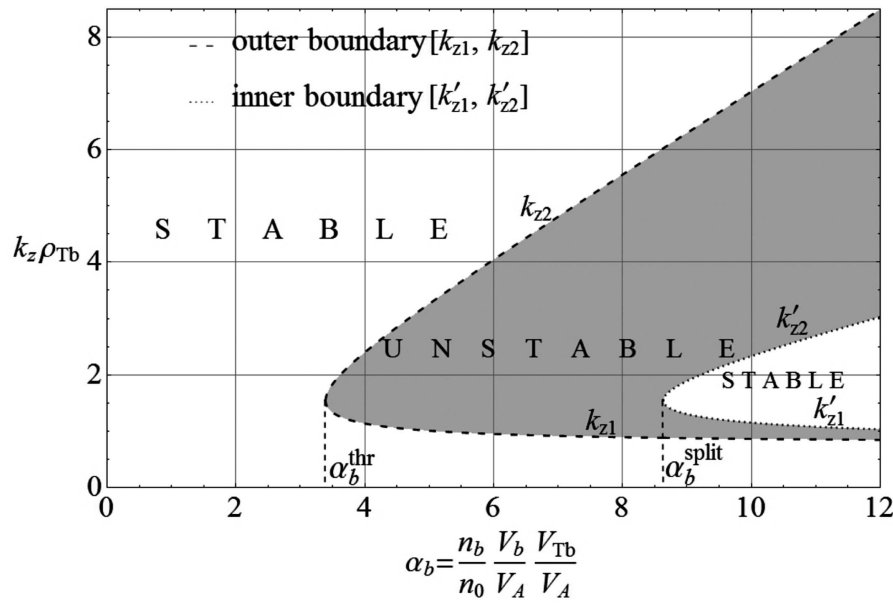

Fig. 4. Unstable wavenumber ranges in the $\left(\alpha_{\mathrm{b}}, k_{\mathrm{z}}\right)$ plane for $V_{\mathrm{A}} / V_{\mathrm{b}}=$ 0.9 . The outer boundary is defined by the left-hand side and the inner boundary by the right-hand side of condition (18). It is seen that below $\alpha_{\mathrm{b}}^{\text {thr }}$ there is no instability, at $\alpha_{\mathrm{b}}^{\text {thr }}<\alpha_{\mathrm{b}}<\alpha_{\mathrm{b}}^{\text {split }}$ there is a single unstable range of $k_{\mathrm{z}}$, and above $\alpha_{\mathrm{b}}^{\text {split }}$ there are two unstable ranges.

$k_{\mathrm{z} 1}^{\prime} \rho_{\mathrm{Tb}} \approx \frac{1}{0.64+\sqrt{0.41-\frac{2}{\alpha_{\mathrm{b}}} \frac{V_{\mathrm{b}}^{2}}{V_{\mathrm{A}}^{2}}\left(1+\sqrt{1-\frac{V_{\mathrm{A}}^{2}}{V_{\mathrm{b}}^{2}}}\right)}}$.

From these expressions we see that with increasing $\alpha_{\mathrm{b}}$ the difference between $k_{\mathrm{z} 1}$ and $k_{\mathrm{z} 1}^{\prime}$ decreases, $k_{\mathrm{z} 1}^{\prime} \rho_{\mathrm{Tb}} \rightarrow k_{\mathrm{z} 1} \rho_{\mathrm{Tb}} \rightarrow 0.766$, and the first unstable range becomes very narrow.

On the other hand, the roots $k_{\mathrm{z} 2} \rho_{\mathrm{Tb}}$ and $k_{\mathrm{z} 2}^{\prime} \rho_{\mathrm{Tb}}$ bounding the second unstable range, are located above $k_{\mathrm{z}}^{*} \rho_{\mathrm{Tb}} \approx 1.54$, where $\zeta_{\mathrm{b}}>-0.65$. Then, using the small argument expansion (7) for $\operatorname{Re} J_{+}\left(\zeta_{\mathrm{b}}\right)$, we find

$k_{\mathrm{z} 2}^{\prime} \rho_{\mathrm{Tb}}=\frac{\alpha_{\mathrm{b}}}{2} \frac{V_{\mathrm{A}}^{2}}{V_{\mathrm{b}}^{2}}\left(1+\sqrt{1-\frac{V_{\mathrm{A}}^{2}}{V_{\mathrm{b}}^{2}}}\right)^{-1}-\frac{2}{\alpha_{\mathrm{b}}} \frac{V_{\mathrm{b}}^{2}}{V_{\mathrm{A}}^{2}}\left(1+\sqrt{1-\frac{V_{\mathrm{A}}^{2}}{V_{\mathrm{b}}^{2}}}\right) ;$

$k_{\mathrm{z} 2} \rho_{\mathrm{Tb}}=\frac{\alpha_{\mathrm{b}}}{2}\left(1+\sqrt{1-\frac{V_{\mathrm{A}}^{2}}{V_{\mathrm{b}}^{2}}}\right)-\frac{2}{\alpha_{\mathrm{b}}}\left(1+\sqrt{1-\frac{V_{\mathrm{A}}^{2}}{V_{\mathrm{b}}^{2}}}\right)^{-1}$.

At large values of $\alpha_{\mathrm{b}}$, both the width of the second unstable range $k_{\mathrm{z} 2} \rho_{\mathrm{Tb}}-k_{\mathrm{z} 2}^{\prime} \rho_{\mathrm{Tb}}$ and the gap between the unstable ranges $k_{\mathrm{z} 2}^{\prime} \rho_{\mathrm{Tb}}-$ $k_{\mathrm{z} 1}^{\prime} \rho_{\mathrm{Tb}}$ grow linearly with $\alpha_{\mathrm{b}}$.

In summary, the most important analytical result obtained here is the instability boundary $\alpha_{\mathrm{b}}^{\text {thr }}$ in the parameter space $\left(V_{\mathrm{A}} / V_{\mathrm{b}} ; \alpha_{\mathrm{b}}\right)$, which can be used directly to analyze observational data. The compensated-current systems with $V_{\mathrm{A}} / V_{\mathrm{b}}<1$ and $\alpha_{\mathrm{b}}>\alpha_{\mathrm{b}}^{\text {thr }}$ are unstable. The unstable area in the parameter space $\left(V_{\mathrm{A}} / V_{\mathrm{b}} ; \alpha_{\mathrm{b}}\right)$ is divided further by $\alpha_{\mathrm{b}}^{\text {split }}$ into two unstable sub-areas: $\alpha_{\mathrm{b}}^{\text {thr }}<\alpha_{\mathrm{b}}<\alpha_{\mathrm{b}}^{\text {split }}$ with one unstable wavenumber range, and $\alpha_{\mathrm{b}}>\alpha_{\mathrm{b}}^{\text {split }}$ with two unstable wavenumber ranges.

\subsection{Instability growth rate}

Once $\alpha_{\mathrm{b}}$ rises above $\alpha_{\mathrm{b}}^{\text {thr }}$, an unstable range between $k_{\mathrm{z} 1}$ and $k_{\mathrm{z} 2}$ appears. The instability growth rate (14) as function of $k_{\mathrm{z}}$ is shown in Fig. 5. The plasma parameters $\alpha_{\mathrm{b}}$ and $V_{\mathrm{A}} / V_{\mathrm{b}}$ in this 


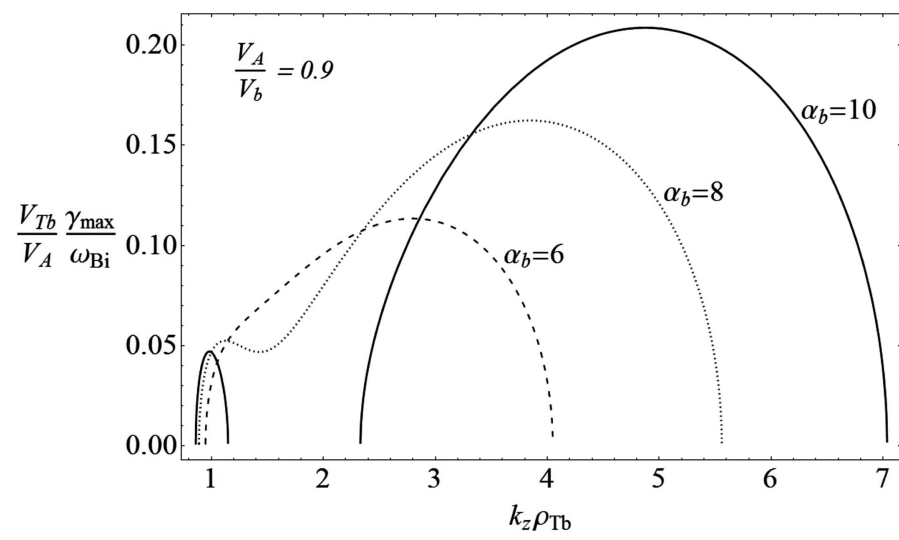

Fig. 5. Wavenumber dependence of the instability growth rate driven by super-Alfvénic ion beams with $V_{\mathrm{A}} / V_{\mathrm{b}}=0.9$ for three values of $\alpha_{\mathrm{b}}$ : $\alpha_{\mathrm{b}}=6,8$, and 10. For larger $\alpha_{\mathrm{b}}$, the unstable area and the maximum growth rate extend to larger $k_{\mathrm{z}} \rho_{\mathrm{Tb}}$.

figure are chosen in such a way as to illustrate the behavior of CCPI in the unstable wavenumber ranges found above. So, the case $\alpha_{\mathrm{b}}=6$ with one unstable wavenumber range is shown by the dashed line and the case $\alpha_{\mathrm{b}}=10$ with two unstable wavenumber ranges is shown by the solid lines. The dotted curve in Fig. 5 is for the case $\alpha_{\mathrm{b}}=8$, which is close to the splitting threshold. It is seen that when the right instability boundary in (18) approaches the maximum of function $\left(k_{\mathrm{z}} \rho_{\mathrm{Tb}}\right)^{-1} A_{\mathrm{k}}$, the valley and the second peak in $\gamma_{\mathrm{k}}$ appear. This happens at $\alpha_{\mathrm{b}}>\alpha_{\mathrm{b}}^{\mathrm{pl}}$, where

$\alpha_{\mathrm{b}}^{\mathrm{pl}} \approx \frac{V_{\mathrm{b}}^{2}}{V_{\mathrm{A}}^{2}}\left(4.1+\sqrt{16-15 \frac{V_{\mathrm{A}}^{2}}{V_{\mathrm{b}}^{2}}}\right)$

is the value of $\alpha_{\mathrm{b}}$ at which a local "plateau" in $\gamma_{\mathrm{k}}$ occurs at the wavenumber where $\partial \gamma_{\mathrm{k}} / \partial k_{\mathrm{z}}=0$ and $\partial^{2} \gamma_{\mathrm{k}} / \partial k_{\mathrm{z}}^{2}=0$. For all $\alpha_{\mathrm{b}}>\alpha_{\mathrm{b}}^{\mathrm{pl}}$, the secondary peak of $\gamma_{\mathrm{k}}$ exists at $k_{\mathrm{z}}<k_{\mathrm{z}}^{*}$. Since $\alpha_{\mathrm{b}}^{\mathrm{pl}}<\alpha_{\mathrm{b}}^{\text {split }}$, the secondary peak arises before the interval of prohibited wavenumbers $k_{\mathrm{z} 1}^{\prime}<k_{\mathrm{z}}<k_{\mathrm{z} 2}^{\prime}$ appears.

It is seen that CCPI is stronger and the most unstable wavenumbers are larger for larger $\alpha_{\mathrm{b}}$. The secondary peak that appears at $\alpha_{\mathrm{b}}>\alpha_{\mathrm{b}}^{\mathrm{pl}}$ is lower than the main peak. These trends are confirmed below analytically.

The most unstable wavenumber and the corresponding maximum growth rate $\gamma_{\max }$ can be found by maximizing (14) with respect to $k_{\mathrm{z}}, \gamma_{\max }=\max _{k}\left(\gamma_{\mathrm{k}}\right)$, which we call the CCPI growth rate. The normalized CCPI growth rate $\gamma_{\max } / \omega_{\mathrm{Bi}}$ as a function of $n_{\mathrm{b}} / n_{0}$ and $V_{\mathrm{b}} / V_{\mathrm{A}}$ is shown in Fig. 6 for hot beam with $V_{\mathrm{Tb}} / V_{\mathrm{A}}=10^{2}$. It is seen that $\gamma_{\max }$ increases rapidly, roughly proportional to both $n_{\mathrm{b}} / n_{0}$ and $V_{\mathrm{b}} / V_{\mathrm{A}}$, which means it is proportional to the current $n_{\mathrm{b}} V_{\mathrm{b}}$. This behavior agrees with the current nature of CCPI confirmed below analytically by (34) and (35).

The threshold for $n_{\mathrm{b}} / n_{0}\left(V_{\mathrm{b}} / V_{\mathrm{A}}\right)$ is lower for smaller $V_{\mathrm{b}} / V_{\mathrm{A}}$ $\left(n_{\mathrm{b}} / n_{0}\right)$, in agreement with (19). In particular, the velocity threshold $V_{\mathrm{b}}^{\mathrm{thr}} / V_{\mathrm{A}}$ decreases with $n_{\mathrm{b}} / n_{0}$ and reaches the minimum value $V_{\mathrm{b}}^{\mathrm{thr}} / V_{\mathrm{A}} \rightarrow 1$ when $n_{\mathrm{b}} V_{\mathrm{Tb}} /\left(n_{0} V_{\mathrm{A}}\right) \rightarrow 1$.

Dependence of $\gamma_{\max }$ on the thermal velocity $V_{\mathrm{Tb}}$ is somewhat different (see Fig. 7). First, near the threshold, $\gamma_{\max }$ increases very rapidly with $V_{\mathrm{Tb}}$. But this increase quickly slows down as $V_{\mathrm{Tb}}$ departs further from the threshold. Already at $V_{\mathrm{Tb}} \gtrsim 3 V_{\mathrm{Tb}}^{\mathrm{thr}}$, $\gamma_{\max }$ becomes virtually independent of $V_{\mathrm{Tb}}$.

To understand this behavior, we proceed with the analytical analysis. Here we take into account that in the wavenumber

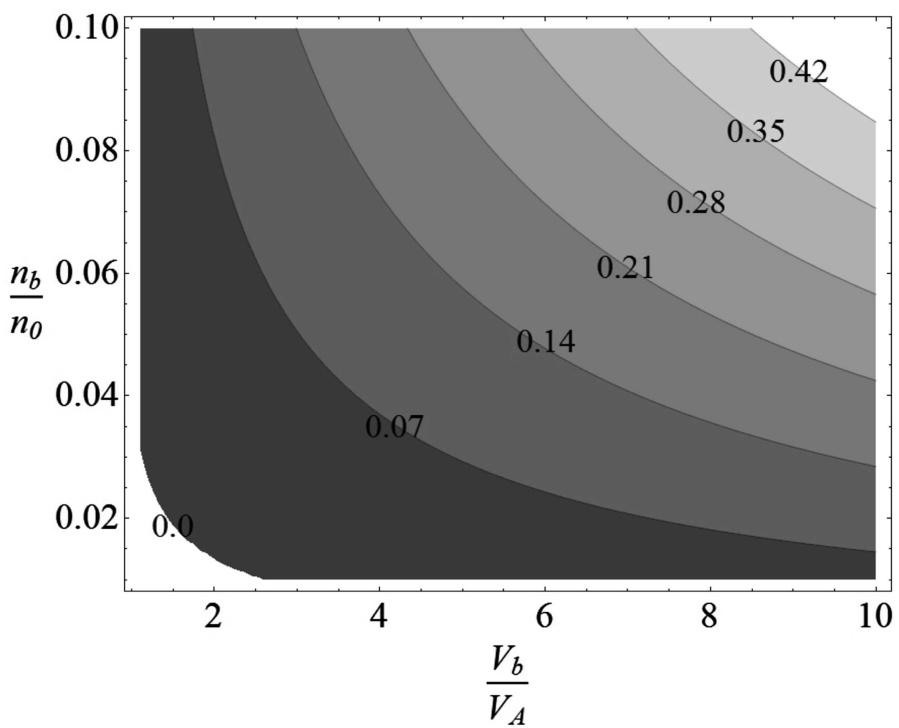

Fig. 6. Normalized growth rate $\gamma_{\max } / \omega_{\mathrm{Bi}}$ as a function of $n_{\mathrm{b}} / n_{0}$ and $V_{\mathrm{b}} / V_{\mathrm{A}}$ for hot beam with $V_{\mathrm{Tb}} / V_{\mathrm{A}}=10^{2} \cdot \gamma_{\max }$ increases regularly with both $n_{\mathrm{b}} / n_{0}$ and $V_{\mathrm{b}} / V_{\mathrm{A}}$ once the threshold is exceeded.

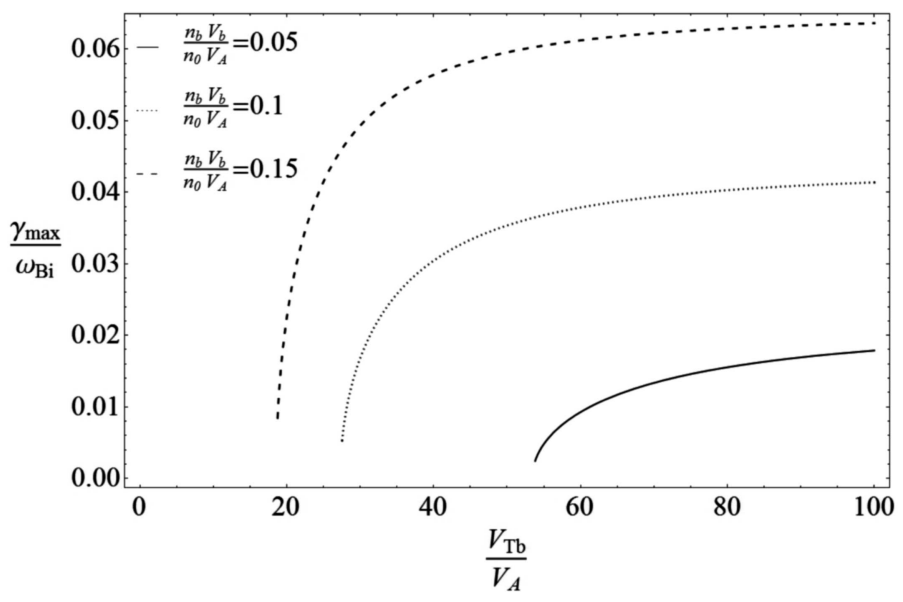

Fig. 7. Normalized growth rate $\gamma_{\max } / \omega_{\mathrm{Bi}}$ as a function of $V_{\mathrm{Tb}} / V_{\mathrm{A}}$ for $n_{\mathrm{b}} V_{\mathrm{b}} /\left(n_{0} V_{\mathrm{A}}\right)=0.05$ ( solid curve), 0.1 (dotted curve), and 0.15 (dashed curve). Starting from zero, the growth rate $\gamma_{\max }$ increases rapidly with $V_{\mathrm{Tb}}$, but this increase is quickly saturated. Larger currents $n_{\mathrm{b}} V_{\mathrm{b}} /\left(n_{0} V_{\mathrm{A}}\right)$ result in larger $\gamma_{\max }$ for all $V_{\mathrm{Tb}}$.

range $k_{\mathrm{z}} \rho_{\mathrm{Tb}}>k_{\mathrm{z}}^{*} \rho_{\mathrm{Tb}} \approx 1.54$, where the growth rate attains its maximum, the low- $\zeta_{\mathrm{b}}$ approximation $\operatorname{Re} J_{+}\left(\zeta_{\mathrm{b}}\right) \approx-\left(k_{\mathrm{z}} \rho_{\mathrm{Tb}}\right)^{-2}$ is valid. Thus, using $A_{\mathrm{k}}=1+\operatorname{Re} J_{+}\left(\zeta_{\mathrm{b}}\right) \approx 1-\left(k_{\mathrm{z}} \rho_{\mathrm{Tb}}\right)^{-2}$ in $(16)$, we find the following approximation for the maximum of $\gamma_{\mathrm{k}}$ :

$\frac{\gamma_{\max }}{\omega_{\mathrm{Bi}}} \approx \frac{1}{2} \frac{n_{\mathrm{b}} V_{\mathrm{b}}}{n_{0} V_{\mathrm{A}}} \sqrt{\left(1-\frac{V_{\mathrm{A}}^{2}}{V_{\mathrm{b}}^{2}}\right)\left[1-\left(\frac{\alpha_{\mathrm{b}}^{\mathrm{thr}}}{\alpha_{\mathrm{b}}}\right)^{2}\right]}$.

This maximum occurs at

$k_{\mathrm{z}}^{\mathrm{m}} \rho_{\mathrm{Tb}} \approx \frac{\alpha_{\mathrm{b}}}{2}+\frac{V_{\mathrm{b}}}{V_{\mathrm{Tb}}} \frac{2}{\alpha_{\mathrm{b}}}\left(1-2 \frac{V_{\mathrm{A}}^{2}}{V_{\mathrm{b}}^{2}}\right)$.

The last term in the square parentheses in (31) is adjusted by replacing the approximate numerical value $\sqrt{8}$ by $\alpha_{\mathrm{b}}^{\text {thr }}$ to make it compatible with the exact $\alpha_{\mathrm{b}}$ threshold (19). We verified numerically that the approximation (31) is good for arbitrary $\alpha_{\mathrm{b}}$, both 
near the threshold and far from it. In general, with the larger beam velocity and/or temperature, the smaller beam density is needed for instability.

The explicit dependence of the instability growth rate on the beam thermal velocity $\bar{V}_{\mathrm{Tb}}$ follows from (31):

$$
\frac{\gamma_{\max }}{\omega_{\mathrm{Bi}}} \approx \frac{\bar{j}_{\mathrm{b}}}{2} \sqrt{\left(1-\frac{1}{\bar{V}_{\mathrm{b}}^{2}}\right)\left[1-\left(\frac{\alpha_{\mathrm{b}}^{\mathrm{thr}}}{\bar{j}_{\mathrm{b}} \bar{V}_{\mathrm{Tb}}}\right)^{2}\right]} .
$$

It is seen that $\gamma_{\max }$ increases quickly with $\bar{V}_{\mathrm{Tb}}$ once the threshold is overcome, $\bar{V}_{\mathrm{Tb}} \gtrsim \alpha_{\mathrm{b}}^{\mathrm{thr}} / \bar{j}_{\mathrm{b}}$. The fast increase of $\gamma_{\max }$ reflects the instability response to the progressive demagnetization of the beam ions as their velocity spread increases above the threshold.

However, when $\bar{V}_{\mathrm{Tb}}$ becomes large enough, $\bar{V}_{\mathrm{Tb}} \gtrsim 3 \alpha_{\mathrm{b}}^{\mathrm{thr}} / \bar{j}_{\mathrm{b}}$, the term containing it becomes negligibly small and $\gamma_{\max }$ becomes virtually independent of $\bar{V}_{\mathrm{Tb}}$. In this high-temperature regime the beam ions are fully demagnetized and the further increase of $\bar{V}_{\mathrm{Tb}}$ no longer affects the instability. This regime corresponds to the asymptotic over-threshold limit $\left(\alpha_{\mathrm{b}}^{\mathrm{thr}} / \alpha_{\mathrm{b}}\right)^{2} \ll 1$, where $\gamma_{\max }$ simplifies to

$\frac{\gamma_{\mathrm{max}}}{\omega_{\mathrm{Bi}}}=\frac{1}{2} \frac{n_{\mathrm{b}} V_{\mathrm{b}}}{n_{0} V_{\mathrm{A}}} \sqrt{1-\frac{V_{\mathrm{A}}^{2}}{V_{\mathrm{b}}^{2}}}$.

The familiar threshold velocity of the beam, $V_{\mathrm{b}}^{\mathrm{thr}}=V_{\mathrm{A}}$, is still present in (34), but the temperature dependence is already missed, as can be observed in Fig. 7 at large $V_{\mathrm{Tb}}$.

The maximum growth rate (34) simplifies further for the fast beams with $V_{\mathrm{b}} / V_{\mathrm{A}}>3$,

$\frac{\gamma_{\max }}{\omega_{\mathrm{Bi}}} \approx \frac{1}{2} \frac{n_{\mathrm{b}} V_{\mathrm{b}}}{n_{0} V_{\mathrm{A}}}$

with the most unstable parallel wavenumber $k_{\mathrm{z}}^{\mathrm{m}} \rho_{\mathrm{Tb}}=\alpha_{\mathrm{b}} / 2$. The asymptotic scaling (35) recovers the scaling obtained by Bell (2004). As is seen from Fig. 7, expressions (34) and (35) provide good estimations for $\gamma_{\max }$ at $V_{\mathrm{Tb}}>3 V_{\mathrm{Tb}}^{\mathrm{thr}}$, which also quantifies the meaning of "asymptotic regime" in terms of $V_{\mathrm{Tb}}$. It appears that the expressions found by Bell are only valid in this asymptotic regime.

For $\alpha_{\mathrm{b}}>\alpha_{\mathrm{b}}^{\mathrm{pl}}$, the secondary peak arises at $k_{\mathrm{z}} \rho_{\mathrm{Tb}}<1.54$, where we can use approximation (25). Then for this peak we obtain the local maximum

$$
\frac{\gamma_{\mathrm{m} 2}}{\omega_{\mathrm{Bi}}} \approx \frac{V_{\mathrm{A}}}{V_{\mathrm{Tb}}} k_{\mathrm{z}}^{\mathrm{m} 2} \rho_{\mathrm{Tb}} \sqrt{\left(1-\frac{V_{\mathrm{A}}^{2}}{V_{\mathrm{b}}^{2}}\right)}
$$

attained at

$k_{\mathrm{z}}^{\mathrm{m} 2} \rho_{\mathrm{Tb}} \approx 0.765+\frac{2}{\alpha_{\mathrm{b}}^{*}}$,

where $\alpha_{\mathrm{b}}^{*}=\left(\frac{V_{\mathrm{A}}}{V_{\mathrm{b}}}\right)^{2} \alpha_{\mathrm{b}}$ and we took into account that $\alpha_{\mathrm{b}}>\alpha_{\mathrm{b}}^{\mathrm{pl}}$. The ratio of this peak to the main peak is

$\frac{\gamma_{\mathrm{m} 2}}{\gamma_{\max }}=\frac{k_{\mathrm{z}}^{\mathrm{m} 2} \rho_{\mathrm{Tb}}}{\frac{1}{2} \alpha_{\mathrm{b}}}=\left(\frac{V_{\mathrm{A}}}{V_{\mathrm{b}}}\right)^{2}\left(0.765+\frac{2}{\alpha_{\mathrm{b}}^{*}}\right) \frac{2}{\alpha_{\mathrm{b}}^{*}}$.

Taking into account that $\alpha_{\mathrm{b}}^{*}>\min \left[\alpha_{\mathrm{b}}^{*}\right] \approx 5.1\left(\right.$ at $V_{\mathrm{A}} / V_{\mathrm{b}} \rightarrow 1$ ), we see that the peak $\gamma_{\mathrm{m} 2}$ is always significantly smaller than the main peak $\gamma_{\max }$. The maximum ratio $\gamma_{\mathrm{m} 2} / \gamma_{\max } \approx 0.45$ is achieved at $\alpha_{\mathrm{b}} \gtrsim \alpha_{\mathrm{b}}^{\mathrm{pl}}$ and $V_{\mathrm{A}} / V_{\mathrm{b}} \lesssim 1$.
We note that the unstable fluctuations also have a small oscillatory part $\operatorname{Re}[\omega]=0.5\left(n_{\mathrm{b}} / n_{0}\right) \omega_{\mathrm{Bi}}$. For most unstable wavenumber $k_{\mathrm{z}}^{\mathrm{m}} \rho_{\mathrm{Tb}} \sim \alpha_{\mathrm{b}} / 2$, the real frequency $\omega^{\mathrm{m}}=\operatorname{Re}[\omega] \sim k_{\mathrm{z}}^{\mathrm{m}} V_{\mathrm{A}}\left(V_{\mathrm{A}} / V_{\mathrm{b}}\right)$ is smaller than the frequency of the normal Alfvén mode $k_{\mathrm{z}}^{\mathrm{m}} V_{\mathrm{A}}$. Since $\gamma_{\max } \sim k_{\mathrm{z}}^{\mathrm{m}} V_{\mathrm{A}}>\omega^{\mathrm{m}}$, the instability is aperiodic.

\section{Parallel Alfvén instability in particular compensated-current systems}

Let us consider two feasible applications of CCPI. First we apply our results to the solar wind upstream of the quasi-parallel terrestrial shock where the plasma conditions are relatively well documented. Then we extend the analysis to the interstellar medium around supernova remnants, assuming the similar scalings of the beam parameters as in the terrestrial foreshock.

\subsection{Quasi-parallel terrestrial foreshock}

Hot ion beams with $V_{\mathrm{Tb}}>V_{\mathrm{b}}>V_{\mathrm{A}}$ are regularly observed in the solar wind upstream of the terrestrial bow shock where the shock normal is quasi-parallel to the interplanetary magnetic field $\boldsymbol{B}_{0}$ (Paschmann et al. 1981; Tsurutani \& Rodriguez 1981). This ordering of characteristic velocities suggests that CCPI driven by hot ion beams can develop in the quasi-parallel foreshocks.

More specifically, we use the following scalings for characteristic beam velocities, $V_{\mathrm{b}} \lesssim V_{\text {shock }}$ and $V_{\mathrm{Tb}} \sim 3 V_{\text {shock}}$, where the shock velocity is equal to the solar wind speed, $V_{\text {shock }}=V_{\mathrm{SW}}$. These scalings are compatible with observations reported by Paschmann et al. (1981) and Tsurutani \& Rodriguez (1981). Yet another beam parameter, number density $n_{\mathrm{b}}$, does not vary much around $n_{\mathrm{b}}=0.1 \mathrm{~cm}^{-3}$ (Paschmann et al. 1981). In terms of the background solar-wind density $n_{0} \sim 5-10 \mathrm{~cm}^{-3}$, this gives $n_{\mathrm{b}} / n_{0} \sim 0.01-0.02$. Taking the typical value of Alfvén velocity, $V_{\mathrm{A}} \approx 0.1 V_{\mathrm{SW}}$, we obtain the cumulative destabilizing parameter $\alpha_{\mathrm{b}} \sim 2.5-5$, which is slightly over-threshold depending on the particular value of $V_{\mathrm{b}}$. Such proximity of the system to the CCPI threshold can be a signature of CCPI operating in the foreshock and relaxing the beam parameters towards the threshold.

On the other hand, as is seen from Fig. 6, even slight deviations of $\alpha_{\mathrm{b}}$ from the threshold can make CCPI strong. So, for $V_{\mathrm{A}} / V_{\mathrm{b}} \sim 0.1$ and $\alpha_{\mathrm{b}}=6$ the maximum growth rate is already high, $\gamma_{\max } \approx 0.07 \omega_{\mathrm{Bi}}$, with the most unstable wavenumbers $k_{z \mathrm{~m}} \rho_{\mathrm{Tb}} \gtrsim 2$. Narita et al. (2006) and Hobara et al. (2007) analyzed properties of electromagnetic fluctuations observed around terrestrial bow shock. Most straightforwardly, our results can be compared with the wavenumber distribution of the fluctuations in the quasi-parallel foreshocks shown in Fig. 9 by Narita et al. (2006), where the measured wavenumbers are normalized by the ion gyroradius. In terms of the background ion gyroradius $\rho_{T i}$, with the typical temperature of the diffuse ions $T_{\mathrm{b}} / T_{\mathrm{i}}=4 \times 10^{2}$, our most unstable wavenumbers $k_{z \mathrm{~m}} \rho_{T i} \sim k_{z \mathrm{~m}} \rho_{\mathrm{Tb}} / 20 \sim 0.1$ map upon the major peak observed at $k_{\mathrm{z}} \rho_{T i}=0.1$ (see upper panel of Fig. 9 in Narita et al. 2006).

In the quasi-parallel foreshock region, Narita et al. observed another, subdominant peak at $k_{\mathrm{z}} \rho_{T i}=0.6$. To explain this peak by CCPI we need a significantly lower beam temperature, $T_{\mathrm{b}} / T_{\mathrm{i}} \sim 10$, which is more typical for quasi-perpendicular foreshocks. We can speculate that CCPI can also generate this second peak. First, the CCP instability develops in the quasiperpendicular foreshock region where the beams have required temperatures $T_{\mathrm{b}} / T_{\mathrm{i}} \sim 10$, which is supported by the observed 
enhancement at $k_{\mathrm{z}} \rho_{T \mathrm{i}} \approx 0.4$. Then the unstable fluctuations are convected in the quasi-parallel foreshock region where their observed wavenumbers are $k_{\mathrm{z}} \rho_{T \mathrm{i}} \approx 0.6$.

The above estimations suggest that CCPI can contribute to electromagnetic fluctuations observed in the quasi-parallel terrestrial foreshock and can impose limitations on the parameters of the beams formed by reflected ions. Further direct confrontations of observed values of $\alpha_{\mathrm{b}}$ with the stability diagram in Fig. 1 are needed to clarify the role of CCPI in the regulation of ionbeam parameters in the foreshock.

\subsection{Foreshock regions around supernova remnants}

Supernova remnants expanding in the interstellar medium develop bow shocks at their boundaries. These shocks propagate with high velocities $V_{\text {shock }} \sim 2 \times 10^{9} \mathrm{cms}^{-1}$ providing a feasible source of energy for the cosmic ray acceleration, and also for the magnetic field amplification. By analogy with the terrestrial bow shock, we assume that the reflected ions also occur in the supernova foreshocks setting up a compensated-current system. CCPI can develop in supernova foreshocks if parameters of reflected ions (subscript b) satisfy $\alpha_{\mathrm{b}}>\alpha_{\mathrm{b}}^{\text {thr }}$, defined by (19).

For reasonable background density $n_{0}=10^{-2}-1 \mathrm{~cm}^{-3}$ and magnetic field $B_{0} \sim 10^{-7}-10^{-5} \mathrm{G}$ (Zweibel \& Everett 2010), the Alfvén velocity varies in the range $V_{\mathrm{A}}=2 \times 10^{4}-2 \times 10^{7} \mathrm{~cm} / \mathrm{s}$. Then the resulting Alfvén Mach number in supernova remnants $M_{\mathrm{A}}=V_{\text {shock }} / V_{\mathrm{A}}=10^{2}-10^{5}$ is much larger than in Earth's bow shock. For the similar scalings as in the terrestrial foreshocks, $n_{\mathrm{b}} / n_{0} \sim 0.01, V_{\mathrm{b}} \sim 0.5 V_{\text {shock }}$, and $V_{\mathrm{Tb}} \sim 2 V_{\text {shock }}$, even with the most unfavorable $V_{\text {shock }} / V_{\mathrm{A}}=10^{2}$ the destabilizing parameter $\alpha_{\mathrm{b}} \sim 10^{2}$ is much larger than the threshold $\alpha_{\mathrm{b}}^{\text {thr }} \sim 5$. In this far over-threshold range, the CCPI operates in the asymptotic regime (35) with very high growth rate $\gamma_{\max } / \omega_{\mathrm{Bi}} \sim 0.5$. We note that this value is already at the edge of applicability of our low-frequency approximation. Such a high growth rate suggests that the instability modifies the beam parameters strongly, in particular reducing the beam velocity towards the local Alfvén velocity, $V_{\mathrm{b}} \gtrsim V_{\mathrm{A}}$.

Let us compare the instability driven by the reflected ions with the similar instability driven by cosmic rays around supernova remnants (Bell 2004; Zweibel \& Everett 2010). Taking the background magnetic field $B_{0} \gtrsim 10^{-6} \mathrm{G}$ and the cosmicrays flux $n_{\mathrm{CR}} V_{\mathrm{b}} \sim 10^{4} \mathrm{~cm}^{-2} \mathrm{~s}^{-1}$ (Zweibel \& Everett 2010), we estimate the normalized current $\bar{j}_{\mathrm{b}}^{\mathrm{CR}} \sim 0.026$ and the corresponding growth rate $\gamma_{\max }^{\mathrm{CR}} / \omega_{\mathrm{Bi}}=\bar{j}_{\mathrm{b}}^{\mathrm{CR}} / 2 \sim 0.01$ around supernova remnants. With $\omega_{\mathrm{Bi}} \simeq 0.03 \mathrm{~s}^{-1}$, we get $\gamma_{\max }^{\mathrm{CR}} \simeq 2.2 \times 10^{-4} \mathrm{~s}^{-1}$ in absolute numbers.

The above estimations show that the CCPI instability driven by reflected ions is much stronger than the instability driven by cosmic rays. Therefore, the former instability can be a more efficient amplifier for magnetic fields around supernova remnants. On the other hand, a fraction of the beam ions can be scattered back to the shock by electromagnetic fluctuations generated by CCPI, thus providing a seed population for the further Fermi acceleration to high cosmic-ray energies.

\section{Discussion}

A number of competing electrostatic and electromagnetic instabilities may arise when different plasma species move with respect to each other (see Gary 2005; Bret 2009, and references therein). The hierarchical structure of these instabilities depends on many parameters and remains an open question

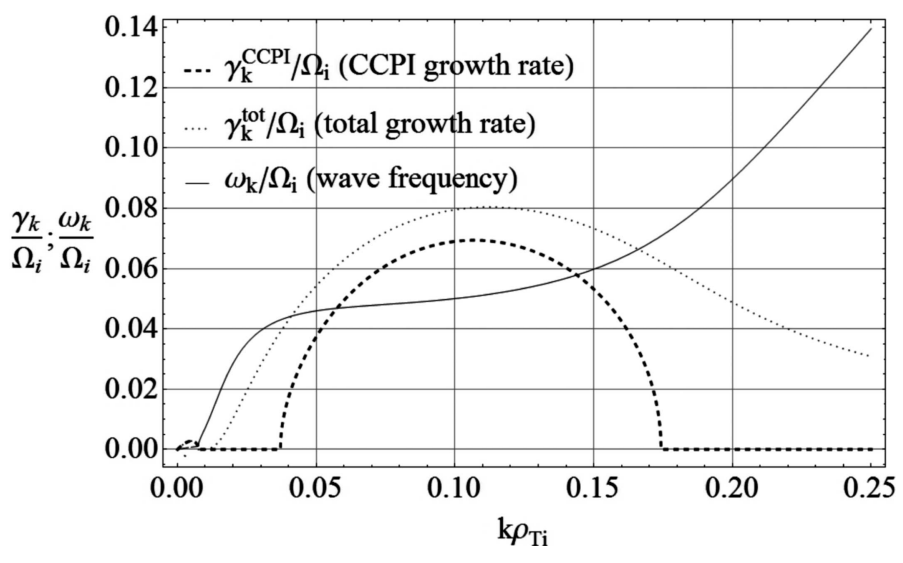

Fig. 8. Contribution of the reactive CCPI growth rate (dashed curve) to the total growth rate (dotted curve) for $V_{T i} / V_{\mathrm{A}}=T_{\mathrm{e}} / T_{\mathrm{i}}=1, V_{\mathrm{b}} / V_{\mathrm{A}}=10$, $V_{\mathrm{Tb}} / V_{\mathrm{A}}=25$, and $n_{\mathrm{b}} / n_{0}=0.02$ : It is seen that the reactive destabilizing effects dominate the instability growth rate for this set of parameters. The wave frequency is shown by the solid line.

(see further discussions in Bret et al. 2010; Brown et al. 2013; Marcowith et al. 2016).

In our setting with hot ion beams, the fast two-stream/ Buneman instabilities are quenched by the large thermal velocities, which are larger than the streaming velocities. Inspection of Fig. 3.20 by Gary (2005) shows that the thresholds of electrostatic ion-acoustic and ion-cyclotron instabilities are significantly higher than the Alfvénic threshold for $V_{T \mathrm{i}} / V_{\mathrm{A}} \sim T_{\mathrm{e}} / T_{\mathrm{i}} \sim 1$ typical in the terrestrial foreshock. Among them, the electron/ion cyclotron instability has the lowest threshold velocity, which is still very high, $V_{\mathrm{b}}^{I C}>10^{2} V_{\mathrm{A}}$ for $n_{\mathrm{b}}<0.1 n_{\mathrm{e}}$. The ion/ion acoustic instability is suppressed further by large beam temperatures, as is seen from Fig. 3.15 by Gary (2005). Therefore, these high-frequency electrostatic instabilities cannot compete with CCPI in the wide range of beam velocities $1<V_{\mathrm{b}} / V_{\mathrm{A}}<10^{2}$. At higher beam velocities, $V_{\mathrm{b}} / V_{\mathrm{A}}>10^{2}$, the ion-acoustic and ion-cyclotron harmonic waves can be generated by the electron-ion relative motion. However, even in this velocity range CCPI can develop independently as long as the mean parameters reside in the unstable area (Fig. 1), whereas the kinetic instabilities are quickly saturated by the local quasi-linear plateaus.

Parallel-propagating left-hand and right-hand polarized instabilities have been studied by Gary et al. (1984) and Gary (1985). Using numerical solutions of the dispersion equation, it has been observed that the left-hand polarized Alfvénic instability becomes competitive or even dominant when the beam ions are sufficiently hot (see Fig. 8 by Gary et al. 1984). The condition $\left|\xi_{\mathrm{b},-1}\right|<1$ was used by Gary et al. to categorize this instability as ion-beam resonant, i.e., resulting from the direct resonant coupling of the unstable mode with the beam ions. However, kinetic and reactive effects have not been distinguished for this mode, which did not allow us to realize that above threshold (19) the instability transforms from kinetic resonant to reactive nonresonant (see Fig. 8 and related discussions below). In the reactive regime, the meaning of the condition $\left|\xi_{\mathrm{b},-1}\right| \approx\left|k_{\mathrm{z}} \rho_{\mathrm{Tb}}\right|^{-1}<1$ is reversed: here it indicates that the unstable perturbations are on small enough scales to decouple from the beam ions by the demagnetization effect. The resulting Alfvén instability is then driven not by the resonant interactions with the beam ions, but by the bulk return current of the magnetized electrons. The current nature of this instability is similar to the nature of related 
current instability (Malovichko \& Iukhimuk 1992) that can develop in the absence of any beams.

Interplay of the reactive and resonant left-hand Alfvénic instabilities also needs further investigations. Our preliminary estimations indicate that the relative importance of the reactive versus kinetic destabilizing effects increases quickly once $\alpha_{\mathrm{b}}$ rises above the threshold $\alpha_{\mathrm{b}}^{\mathrm{thr}}$. In Fig. 8. we show the contribution of the reactive CCPI to the total growth rate for reference plasma parameters that may occur in foreshocks: $V_{T \mathrm{i}} / V_{\mathrm{A}}=T_{\mathrm{e}} / T_{\mathrm{i}}=1$, $V_{\mathrm{b}} / V_{\mathrm{A}}=10, V_{\mathrm{Tb}} / V_{\mathrm{A}}=25$, and $n_{\mathrm{b}} / n_{0}=0.02$. The corresponding total growth rate in Fig. 8 is given by Eq. (14) with the imaginary part of $J_{+}$taken into account. It therefore includes both the reactive effects due to the bulk currents and the resonant waveparticle interactions. It is seen that the destabilizing reactive response becomes stronger than the resonant wave response when $\alpha_{\mathrm{b}}$ is still not far from the threshold $\alpha_{\mathrm{b}}^{\text {thr }}\left(\alpha_{\mathrm{b}}=5 \approx 2 \alpha_{\mathrm{b}}^{\text {thr }}\right.$ in Fig. 8). The instability is thus driven mainly by the reactive effects and can be analyzed ignoring kinetic resonant effects, as we did in the present study. The same approach can also be applied in the immediate vicinity of the reactive threshold if the quasi-linear plateaus or other local deformations of the velocity distributions weaken destabilizing kinetic effects. Analytical treatment becomes more tangled when reactive and kinetic effects are of comparable efficiency and have to be accounted for simultaneously, in which case the evolution of the system becomes more complex (cf. Yoon \& Sarfraz 2017).

There are also left- and right-hand polarized instabilities driven by cold ion beams in the ion-cyclotron frequency range (Mecheri \& Marsch 2007). These instabilities are strong when the velocity spread of the beam ions is so small that all the beam ions (and hence the beam as a whole) are resonant. In our settings with hot ion beams these instabilities are quenched similarly to the two-stream/Buneman instabilities.

In the considered case of hot ion beams, $V_{\mathrm{b}} / V_{\mathrm{Tb}}<1$, the analytical treatment of wavenumbers $k_{\mathrm{z}} \rho_{\mathrm{Tb}}<V_{\mathrm{Tb}} / V_{\mathrm{b}}$ is simplified by neglecting the term $\sim V_{\mathrm{b}} / V_{\mathrm{Tb}}$ in $\xi_{\mathrm{b},-1}$. As the most unstable wavenumber scales as $k_{\mathrm{z}} \rho_{\mathrm{Tb}} \approx \alpha_{\mathrm{b}} / 2$ (32), this restriction is not stringent:

$\frac{n_{\mathrm{b}}}{n_{0}}\left(\frac{V_{\mathrm{b}}}{V_{\mathrm{A}}}\right)^{2}<2$

This condition is the opposite of the firehose instability condition (see Eq. (14) by Malovichko et al. 2014), which means that the CCPI can operate in a wide range of parameters below the firehose threshold. For cooler beams, where the condition $V_{\mathrm{b}} / V_{\mathrm{Tb}}<1$ is violated (e.g., in the quasi-perpendicular foreshock regions), the analysis should be extended by accounting for corresponding terms.

The compensated-current parallel instability can also affect other processes in space. For example, it can limit the field-aligned currents generated by Alfvén-wave fluxes in the inner magnetosphere and plasma sheet boundary layer (Artemyev et al. 2016). In the solar wind, CCPI can contribute to the regulation of relative motion of different plasma species. It was found that many states of beaming structures in the solar wind are close to the thresholds of magnetosonic and Alfvén instabilities (Marsch \& Livi 1987; Gary et al. 2000) and the firehose instability (Chen et al. 2016). Since CCPI can operate close to these thresholds (and sometimes below them), a refined analysis is needed to decide its role in the solar wind, as compared to the magnetosonic and firehose instabilities. These are subjects for future studies.

\section{Conclusions}

We investigated reactive non-resonant compensated-current parallel instability (CCPI) of left-hand polarized Alfvén waves in compensated-current systems established by hot diluted ion beams. Ion-beam demagnetization due to finite $k_{\mathrm{z}} \rho_{\mathrm{Tb}}$ is crucial for CCPI ( $\rho_{\mathrm{Tb}}$ is based on the parallel beam temperature, and thus does not represent the beam ion gyroradius). New analytical expressions for the instability growth rate (31) and threshold (19) are found and analyzed.

The most important new properties of CCPI can be summarized as follows:

1. Reactive non-resonant CCPI depends on all bulk parameters of the beam: beam density $n_{\mathrm{b}}$, bulk velocity $V_{\mathrm{b}}$, and thermal velocity $V_{\mathrm{Tb}}$. These parameters all increase the instability growth rate and can be combined in the single destabilizing parameter $\alpha_{\mathrm{b}}=\left(n_{\mathrm{b}} / n_{0}\right)\left(V_{\mathrm{b}} / V_{\mathrm{A}}\right)\left(V_{\mathrm{Tb}} / V_{\mathrm{A}}\right)$. The instability develops at $\alpha_{\mathrm{b}}>\alpha_{\mathrm{b}}^{\text {thr }}$, where the instability threshold (19) varies from $\alpha_{\mathrm{b}}^{\text {thr }}=2.43$ at $V_{\mathrm{A}} / V_{\mathrm{b}} \rightarrow 0$ to $\alpha_{\mathrm{b}}^{\text {thr }}=4.87$ at $V_{\mathrm{A}} / V_{\mathrm{b}} \rightarrow 1$. The analytical threshold (19) can be directly compared with satellite data to analyze the stability of beam-plasma systems in space.

2. CCPI is strongly affected by the velocity spread of the beam ions $V_{\mathrm{Tb}}$. It defines the range of unstable beam currents, $\bar{j}_{\mathrm{b}} \geq \bar{j}_{\mathrm{b}}^{\mathrm{thr}}$, with the current threshold varying in the range $\bar{j}_{\mathrm{b}}^{\text {thr }}=$ $(2.4-4.9) / \bar{V}_{\mathrm{Tb}}$.

3. The instability growth rate $\gamma_{\max }$ (33) increases sharply with $V_{\mathrm{Tb}}$ once the threshold $V_{\mathrm{Tb}}^{\mathrm{thr}}(20)$ is overcome (Fig. 7). This fast increase is caused by the fast demagnetization of the beam ions, in which case they cannot compensate for the perturbed currents of fully magnetized electrons. In a more distant overthreshold range $\alpha_{\mathrm{b}}>3 \alpha_{\mathrm{b}}^{\text {thr }}$ the temperature dependence weakens because of the nearly saturated demagnetization.

4. From the growth rate $\gamma_{\max }$ (31) it follows that the instability can be strong, $\gamma_{\max } \gtrsim 0.1 \omega_{\mathrm{Bi}}$, even for modest $\alpha_{\mathrm{b}} \lesssim 2 \alpha_{\mathrm{b}}^{\text {thr }}$ not far from the threshold. The most unstable wavenumber $k_{\mathrm{z}} \rho_{\mathrm{Tb}} \gtrsim$ 1.54 near the threshold $\alpha_{\mathrm{b}} \gtrsim \alpha_{\mathrm{b}}^{\text {thr }}$, but increases with $\alpha_{\mathrm{b}}$ quickly approaching the asymptotic scaling $k_{\mathrm{z}} \rho_{\mathrm{Tb}} \sim \alpha_{\mathrm{b}} / 2$. In this asymptotic regime, our growth rate reduces to (35), the same as was obtained by Bell (2004).

5. Two particular applications to the terrestrial foreshocks and supernova remnants show that the reactive CCPI can operate there. An analysis of Sect. 5.2 suggests that the ions reflected from the shocks around supernova remnants can drive stronger instability than the cosmic rays. In the terrestrial foreshock, CCPI can regulate beam parameters generating electromagnetic fluctuations observed at $k_{\mathrm{z}} \rho_{T i} \approx 0.1$.

Our results complement and extend previous studies on electromagnetic instabilities and their role in space and astrophysical plasmas. CCPI can develop around supernova remnants expanding into interstellar medium, participating in the braking process, and heating and redistributing energy in the supernova shocks. The same concerns the solar-wind regions upstream of the terrestrial bow shock, and other heliospheric shocks, where CCPI can bound the beam parameters and contribute to the low-frequency electromagnetic turbulence. Similarly, CCPI can affect other space and astrophysical environments containing super-Alfvénic ion beams and return currents.

Acknowledgements. This research was supported by the Belgian Science Policy Office (through Prodex/Cluster PEA 90316 and IAP Programme project P7/08 CHARM). 


\section{References}

Achterberg, A. 2013, MNRAS, 436, 705

Alexandrov, A. F., Bogdankevič, L. S., \& Rukhadze, A. A. 1984, Principles of Plasma Electrodynamics (Berlin: Springer-Verlag)

Amato, E., \& Blasi, P. 2009, MNRAS, 392, 1591

Artemyev, A. V., Rankin, R., \& Vasko, I. Y. 2016, J. Geophys. Res., 121, 8361

Bell, A. 2004, MNRAS, 353, 550

Bell, A. 2005, MNRAS, 358, 181

Bret, A. 2009, ApJ, 699, 990

Bret, A., Gremillet, L., \& Dieckmann, M. E. 2010, Phys. Plasmas, 17, 120501

Brown, M. R., Browning, P. K., Dieckmann, M. E., Furno, I., \& Intrator, T. P. 2013, Space Sci. Rev., 178, 357

Büchner, J., \& Elkina, N. 2006, Phys. Plasmas, 13, 082304

Chen, L., \& Wu, D. J. 2012, ApJ, 754, 123

Chen, C. H. K., Matteini, L., Schekochihin, A. A. et al. 2016, ApJ, 825, L26

Duijveman, A., Hoyng, P., \& Ionson, J. A. 1981, ApJ, 245, 721

Gary, S. P. 1985, ApJ, 288, 342

Gary, S. P. 2005, Theory of Space Plasma Microinstabilities (Cambridge: Cambridge University Press)

Gary, S. P., Foosland, D. W., Smith, C. W., Lee, M. A., \& Goldstein, M. L. 1984 Phys. Fluids, 27, 1852

Gary, S. P., Yin, L., Winske, D., \& Reisenfeld, D. B. 2000, Geophys. Res. Lett., 27, 1355

Hobara, Y., Walker, S. N., Balikhin, M. et al. 2007, J. Geophys. Res., 112, A07202

Kobzar, O., Niemiec, J., Pohl, M., \& Bohdan, A. 2017, MNRAS, 469, 4985

Malovichko, P. P. 2007, Kinemat. Phys. Celest. Bodies, 23, 185
Malovichko, P. P. 2010, Kinemat. Phys. Celest. Bodies, 26, 62

Malovichko, P. P., \& Iukhimuk, A. K. 1992, Kinematika i Fizika Nebesnykh Tel, 8,20

Malovichko, P., Voitenko, Y., \& De Keyser, J. 2014, ApJ, 780, 175

Malovichko, P., Voitenko, Y., \& De Keyser, J. 2015, MNRAS, 452, 4236

Marcowith, A., Bret, A., Bykov, A. et al. 2016, Rep. Prog. Phys., 79, 046901

Marsch, E. 2006, Liv. Rev. Sol. Phys., 3, 1

Marsch, E., \& Livi, S. 1987, J. Geophys. Res., 92, 7263

Mecheri, R. \& Marsch, E. 2007, A\&A, 474, 609

Narita, Y., Glassmeier, K.-H., \& Fornaçon, K.-H. et al. 2006, J. Geophys. Res., 111, A01203

Paschmann, G., Sckopke, N., \& Papamastorakis, I. et al. 1981, J. Geophys. Res., 86,4355

Sentman, D., Edmiston, J. P., \& Frank, L. A. 1981, J. Geophys. Res., 86, 2039

Schure, K. M., Bell, A. R., O’C Drury, L., \& Bykov, A. M. 2012, Space Sci. Rev., 173, 491

Tsurutani, B., \& Rodriguez, P. 1981, J. Geophys. Res., 86, A6, 4317

Voitenko, Y. 1995, Sol. Phys., 161, 197

Voitenko, Y. 1996, Sol. Phys., 168, 219

Voitenko, Y. \& Pierrard, V. 2015, Sol. Phys., 290, 1231

Vojtenko Y. M., Krishtal' A. N., Kuts S. V., Malovichko P. P., \& Yukhimuk A. K. 1990, Geomagn. Aeron., 30, 901

Winske, D. \& Leroy, M. M. 1984, J. Geophys. Res., 89, 2673

Wu, D. J., Chen, L., \& Wu, C. S. 2012, PhPl, 19, 024511

Yoon, P. H., \& Sarfraz, M. 2017, ApJ, 835, 246

Zhao, J. S., Voitenko, Y., Yu, M. Y., Lu, J. Y., \& Wu, D. J. 2014, ApJ, 793, 107

Zweibel, E. G., \& Everett, J. E. 2010, ApJ, 709, 1412 